\title{
Uma Ontologia de Domínio para a Análise do Diálogo Freireano em Fóruns de Discussão da Educação a Distância
}

\author{
Priscila Barros David ${ }^{1}$, Ernesto Trajano de Lima ${ }^{1}$, Francisco Arquimedes Gadelha \\ Mendes ${ }^{2}$ \\ ${ }^{1}$ Universidade Federal do Ceará - UFC, Instituto UFC Virtual \\ Campus do Pici, Bloco $901,1^{\circ}$ andar \\ CEP: 60.440-554 - Fortaleza - CE - Brasil \\ ${ }^{2}$ Universidade Federal do Ceará - UFC, Centro de Humanidades \\ Campus do Benfica - Av. da Universidade, 2683 \\ CEP: 60.020-180- Fortaleza - CE - Brasil \\ \{priscila, ernesto\}@virtual.ufc.br, arquimedesgadelha@gmail.com
}

\begin{abstract}
This paper presents a computational model based on ontologies to represent the Paulo Freire's concept of dialogue for its subsequent implementation in AVA, with a view to the analysis of interaction processes of distance education. Freire's dialogue is discussed in this paper from a review of the empirical application of its pillars in different contexts and tools. Seeking to encourage the attainment of formative assessments in distance education, is presented, in this research, the development of Freire's dialogue ontology that is the result of an extensive linguistic research which found its main markers in a discussion forum.
\end{abstract}

Resumo. Este artigo apresenta um modelo computacional, baseado em ontologias, para a representação do diálogo freireano e sua posterior implementação em AVA, tendo em vista a análise de processos interacionais da EaD. $O$ diálogo freireano é discutido neste artigo a partir de uma revisão da aplicação empírica de seus pilares em diferentes contextos e ferramentas. Buscando-se favorecer a realização de avaliações formativas na EaD, apresenta-se, nesta pesquisa, o desenvolvimento da ontologia do diálogo freireano, resultado de uma ampla investigação linguística que detectou seus principais marcadores em um fórum de discussão.

\section{Introdução}

A análise de processos interacionais com suporte tecnológico se justifica neste estudo pela relevância que a Educação a Distância (EaD) conquistou no Brasil, ao longo dos últimos anos. De acordo com os dados mais recentes divulgados pelo INEP no Censo da Educação Superior [Brasil 2014], entre 2012 e 2013 as matrículas em cursos de educação superior a distância cresceram $3,6 \%$ e, atualmente, contam com uma participação superior a $15 \%$ nas matrículas da graduação. Embora este crescimento tenha sofrido uma desaceleração em comparação com a primeira década do século XXI, estudar na modalidade a distância já é uma realidade em todo o Brasil.

Como uma das tendências-chave na área de educação aberta e a distância, o Plano Estratégico 2013-2016 do International Council for Open and Distance Education [ICDE 2013] menciona a elevação da qualidade e desempenho dos cursos 
ofertados nesta modalidade, que inclui o desenvolvimento de novas metodologias e tecnologias, para dar suporte à formação profissional mediada por tecnologias digitais.

Em Educação, não se pode falar em qualidade sem se pensar nos modelos de avaliação da aprendizagem aplicados internamente aos processos de formação. Com o propósito de contemplar esta lacuna, encontra-se em desenvolvimento uma pesquisa apoiada financeiramente pelo $\mathrm{CNPq}^{1}$, cujo objetivo consiste em desenvolver um modelo computacional, baseado em ontologias, para implementar um sistema, denominado SAIC, que investiga diferentes facetas da comunicação mediada por computador, com propósitos educacionais: engajamento e interatividade (Conversação); relações sociais estabelecidas entre os atores (Diálogo); tipo de linguagem praticada pelos interlocutores (Textualização); e, por último, as ações voluntárias do emissor da mensagem visando a elaboração de novos conhecimentos (Aprendizagem) [David 2010].

Considerando-se que a categoria Diálogo do SAIC vem sendo refinada ao longo dos últimos anos por meio de estudos empíricos, conforme será discutido na Seção 2, optou-se por iniciar a construção da ontologia de formalização do sistema pelo Diálogo.

Este artigo está, portanto, assim estruturado: na Seção 2, o fundamento teórico do diálogo freireano é apresentado, por meio de uma revisão da aplicação de seus cinco pilares a processos interacionais em cursos a distância; na Seção 3, expõe-se a temática das ontologias, discutindo-se trabalhos relacionados ao que está sendo proposto neste estudo; a Seção 4 descreve o processo de desenvolvimento da ontologia aqui proposta e os resultados parciais da pesquisa; e, finalmente, a Seção 5 apresenta as considerações finais.

\section{Fundamento Teórico}

Todo processo educacional é permeado por uma concepção epistemológica, ou seja, pela forma como se acredita que a aquisição do conhecimento aconteça. A EaD pode, assim, ser compreendida segundo diferentes perspectivas.

A concepção que se defende neste artigo é fortemente inspirada na teoria de Paulo Freire, o qual critica a concepção de educação baseada na transferência de conhecimentos (educação bancária) e argumenta em favor de uma educação dialógica [Freire 2006]. O autor considera que o diálogo instrumentaliza as pessoas, habilitandoas a construírem algo novo, de forma coletiva. Segundo Freire (op cit), o diálogo possui cinco pilares que norteiam a comunicação no contexto educacional: amor, humildade, fé nos homens, esperança e um pensar crítico [Freire 2006].

Em estudos anteriores, realizamos diferentes esforços de aplicação do conceito de diálogo freireano a processos interacionais na Educação a Distância.

O artigo de 2009 foi a primeira tentativa de organizar os pilares do diálogo em categorias aplicáveis a fóruns de discussão [David e Castro-Filho 2009]: i) amor, traduzido em Afetividade, e identificada pela presença de respeito mútuo e proximidade; ii) humildade, traduzida em Simetria Discursiva, e identificada pela

\footnotetext{
${ }^{1}$ Projeto Universal CNPq N ${ }^{\circ}$ 482704/2013-6. Título: Desenvolvimento de Ontologias com Base no Sistema de Análise de Interações Contingentes para a Implementação de Avaliações Formativas em Fóruns de Discussão.
} 
igualdade de papeis entre os participantes (alunos e professor-tutor); iii) fé nos homens, traduzida em Valorização da autonomia e identificada nas mensagens do professor-tutor pelo incentivo à livre expressão por parte dos alunos; iv) esperança, traduzida em Exercício da Autonomia e identificada pelo aprofundamento dos conceitos pelos alunos; e, v) pensar crítico, traduzido em Reflexividade crítica e identificado na demonstração de reflexão quanto ao próprio processo de aprendizagem e/ou dos demais interlocutores.

Os resultados indicaram a manifestação desses parâmetros no fórum mais participativo (29 mensagens) de uma disciplina de graduação a distância, da área de exatas, fomentando um cenário propício ao aprendizado.

Em 2010, um novo estudo foi publicado [David e Castro-Filho 2010], desta vez aplicando-se os parâmetros do Diálogo, em conjunto com as outras categorias do SAIC, a um chat pedagógico de uma disciplina de graduação da área de humanas. Totalizando 288 turnos de conversação, destacou-se no chat a manifestação de Afetividade e Simetria Discursiva. O estudo também ampliou a descrição do parâmetro Reflexividade Crítica, que passou a analisá-la de acordo com três dimensões: i) Reflexividade Crítica Intrapessoal; ii) Reflexividade Crítica Interpessoal; e, iii) Reflexividade Crítica Inexistente.

Em 2011, um novo trabalho foi publicado [Freire et al 2011], aplicando-se os parâmetros do diálogo ao contexto de um curso de formação de professores-tutores da área de exatas, ofertado em nível de extensão. Os resultados apontaram a presença marcante de Afetividade, Simetria Discursiva, Valorização da Autonomia, Exercício da Autonomia e Reflexividade Crítica Interpessoal e Intrapessoal nas 289 mensagens do fórum analisado.

Em 2012, semelhantemente ao estudo de 2010, mais uma vez os parâmetros do diálogo foram aplicados ao fórum de uma disciplina de graduação a distância, desta vez, da área de humanas [David e Castro-Filho 2012]. O fórum analisado totalizou 75 mensagens, das quais apenas 7 foram do professor-tutor. Os resultados indicaram a predominância dos parâmetros Afetividade, Simetria Discursiva e Reflexividade Crítica Interpessoal e Intrapessoal, e também uma boa frequência de Exercício da Autonomia pelos estudantes, a despeito da baixa participação do professor-tutor no fórum.

Em 2013, voltamos a aplicar os parâmetros do diálogo ao contexto da formação de professores-tutores, em uma turma da área de humanas [David et al 2013a]. Neste estudo, duas categorias foram refinadas: Afetividade e Valorização da Autonomia. Na primeira, passou-se a considerar a manifestação parcial e total de afetividade, conforme a presença de um ou dois dos elementos representativos deste parâmetro (respeito mútuo e proximidade). Também passou-se a considerar dois tipos de valorização da autonomia: pelo tutor e pelos pares.

Finalmente, publicamos em 2013 um último estudo no qual os parâmetros do diálogo foram aplicados manualmente a fóruns de discussão da EaD. Em David et al (2013b) realizamos um estudo comparativo da manifestação do diálogo nas duas turmas do curso de formação para professores-tutores, investigadas respectivamente em $2011 \mathrm{e}$ 2013, ligadas às áreas de exatas e de humanas, respectivamente. Os resultados apontaram diferenças quanto à prática da autonomia nos dois contextos (turma de exatas e turma de humanas). 
Todas as investigações em torno do conceito de diálogo freireano apresentadas nesta seção foram executadas manualmente. Compreende-se que para uma ampla validação do SAIC, o qual contém o Diálogo como uma de suas categorias de análise, e também sua futura implementação em AVA, faz-se necessário o uso de técnicas computacionais capazes de automatizar a avaliação da interação na EaD.

Além desta necessidade de automatização, percebeu-se, ainda, que o conhecimento empírico utilizado no processo manual de identificação das categorias do diálogo poderia ser melhor compreendido se fosse formalizado de alguma maneira. Considerando-se estes dois aspectos, optou-se por desenvolver neste estudo uma ontologia para o domínio Diálogo, uma vez que esta técnica computacional permite tanto a formalização do conhecimento quanto a posterior implementação em AVA.

\section{Trabalhos Relacionados}

Nesta seção, apresentaremos dois trabalhos relacionados ao desenvolvimento de ontologias de domínio, ligadas, respectivamente ao campo da Aprendizagem Colaborativa e da Aprendizagem Baseada em Problemas (PBL), cujas metodologias adotadas possuem relação com o que será proposto no presente artigo.

Isotani e Mizoguchi (2007) discutem as dificuldades de se utilizar diversas teorias de aprendizagem que dão suporte à aprendizagem colaborativa durante $\mathrm{o}$ planejamento e análise de atividades em grupo. De acordo com os autores, tal fato se deve à forma complexa e ambígua como essas teorias são descritas. Além disso, cada teoria possui seu próprio foco teórico, nível de profundidade, dentre outros aspectos que precisam ser considerados. Os autores mencionam que para tornar as sessões colaborativas mais eficazes, educadores acreditam que cada participante de um grupo engajado em atividades colaborativas possua um determinado papel, seja ele tutor, tutelado, assistente ou ajudante.

Assim, o estudo de Isotani e Mizoguchi (op cit) propõe-se a construir um modelo baseado em ontologias que auxilie na análise das interações entre indivíduos e no planejamento apropriado de atividades colaborativas para estudantes, oferecendo recomendações baseadas nas teorias de aprendizagem. Nesta perspectiva, utiliza-se de dois modelos (modelo de processos de interação e modelo de aquisição do conhecimento), unificados por meio da construção de uma ontologia, que gerou um novo modelo denominado pelos autores Growth Model Improved by Interaction Paterns (Modelo de Crescimento Estendido por Padrões de Interação) (GMIP). O estudo descreve uma aplicação prática do modelo GIMP: o desenvolvimento de um sistema computacional denominado Concrete and Helpful Ontology-aware Collaborative Learning Authoring Tool (CHOCOLATO). Como resultado, foi possível criar um sistema que dá suporte ao planejamento e análise de atividades colaborativas, fornecendo ajuda ao usuário na escolha de atividades para cada grupo, papéis para cada aluno, estratégias a serem seguidas, além de outras informações que favorecem $o$ planejamento eficaz de sessões colaborativas com justificativas teóricas.

Os autores concluem que a estrutura ontológica desenvolvida apresenta mais um passo em direção ao desenvolvimento de sistemas inteligentes capazes de esclarecer e representar, de forma mais completa, uma teoria de aprendizagem no contexto da aprendizagem colaborativa. Apesar da detalhada descrição do modelo criado, com diversas representações gráficas e exemplo de aplicação prática, o estudo não especifica 
de forma clara as teorias de aprendizagem que foram utilizadas na criação do modelo proposto.

Outro estudo sobre ontologias de domínio que merece destaque é o de Fontes et al (2011). Seu objetivo foi desenvolver uma ontologia capaz de representar os conceitos associados ao campo da Aprendizagem Baseada em Problemas (PBL). Os autores destacam que uma das dificuldades nos trabalhos com a PBL é a ausência de padronização dos conceitos a ela relacionados, o que dificulta a compreensão comum e compartilhada sobre este domínio de conhecimento. Com o objetivo de solucionar esta questão, o artigo propõe uma ontologia que preenche os requisitos de abstração e significação dos conceitos da PBL. A ontologia busca verificar se os estudantes estão cumprindo o ciclo que define a PBL em seis fases: cenário do problema, identificação de fatos, formulação de hipóteses, deficiências de conhecimento, aplicação de novo conhecimento e abstração.

O estudo apresentou como resultado uma ontologia com doze classes, trinta e duas propriedades do tipo object property e quinze propriedades do tipo datatype property. Os autores concluem que a ontologia proposta possibilita definir uma infraestrutura para integrar sistemas inteligentes no nível conceitual do domínio da PBL, além de oferecer vantagens como: compartilhamento, padronização e reuso de informações. A principal lacuna da pesquisa de Fontes et al (op cit) consiste no fato de não especificar todas as classes utilizadas na construção do modelo ontológico. Doze classes são mencionadas, mas somente oito dessas classes são descritas.

\section{Desenvolvimento da Ontologia Proposta}

Nesta seção, descreve-se o estudo proposto neste artigo. Dentre as suas principais contribuições é possível destacar a descrição detalhada do domínio Diálogo (na perspectiva freireana) e como este domínio de conhecimento foi estruturado, formalizado e padronizado para a construção do modelo ontológico. Além disso, as categorias utilizadas serão detalhadas tanto na forma de mapas conceituais (Seção 4.1) quanto na ferramenta de modelagem ontológica (Seção 4.2).

\subsection{O Processo de Construção da Ontologia do Diálogo}

A ontologia do Diálogo foi construída com base na metodologia proposta por Noy e McGuinness (2001), que, em linhas gerais, consiste na delimitação do escopo da ontologia, na identificação dos conceitos do domínio, na transformação destes conceitos em classes da ontologia, na derivação da estrutura hierárquica das classes e no estabelecimento de propriedades das classes da ontologia e de relações entre elas. Este processo tem como ponto de partida um conjunto de perguntas, a saber: (i) Qual o domínio da ontologia? (ii) Qual será o seu uso? (iii) Que tipo de perguntas ela deve responder? (iv) Quem a utilizará?

Considerando tais perguntas, pode-se dizer que a ontologia aqui proposta tem como domínio o Diálogo na perspectiva freireana, na forma apresentada pelo SAIC. Sua utilização principal será auxiliar professores-tutores a identificar interações em fóruns de discussão nas quais o diálogo freireano se manifeste. Para tanto, a ontologia deve ser capaz de responder a perguntas como as seguintes (questões de competência): Considerando uma determinada mensagem, é possível verificar a existência de Diálogo? Que elementos apontam sua existência? Caso não esteja ocorrendo Diálogo, o que pode 
ser feito para que ele passe a acontecer? Como o Diálogo poderia ser promovido ou incentivado?

Estabelecidos o domínio, o seu uso e as questões de competência, foram, então, identificados os conceitos do Diálogo e as relações entre eles. Para possibilitar a representação inicial e a visualização destas informações (conceitos e relações), foram criados mapas conceituais (Figuras 1 e 2).

Na Figura 1, encontram-se ilustradas as categorias do SAIC, em particular o Diálogo, objeto deste artigo. Como apresentado na Seção 2, o Diálogo é composto de cinco parâmetros: Afetividade, Simetria Discursiva, Valorização da Autonomia, Exercício da Autonomia e Reflexividade Crítica. De forma geral, ao analisar uma mensagem, pode-se dizer que, nela, cada um destes parâmetros se apresenta de forma total, parcial ou não se apresenta (ausência). Em alguns casos, entretanto, não existem elementos para classificar a mensagem de forma adequada. Neste caso, diz-se que a classificação é indefinida. Assim, uma determinada mensagem pode possuir, por exemplo, afetividade total, ausência de valorização da autonomia e reflexividade crítica indefinida ${ }^{2}$.

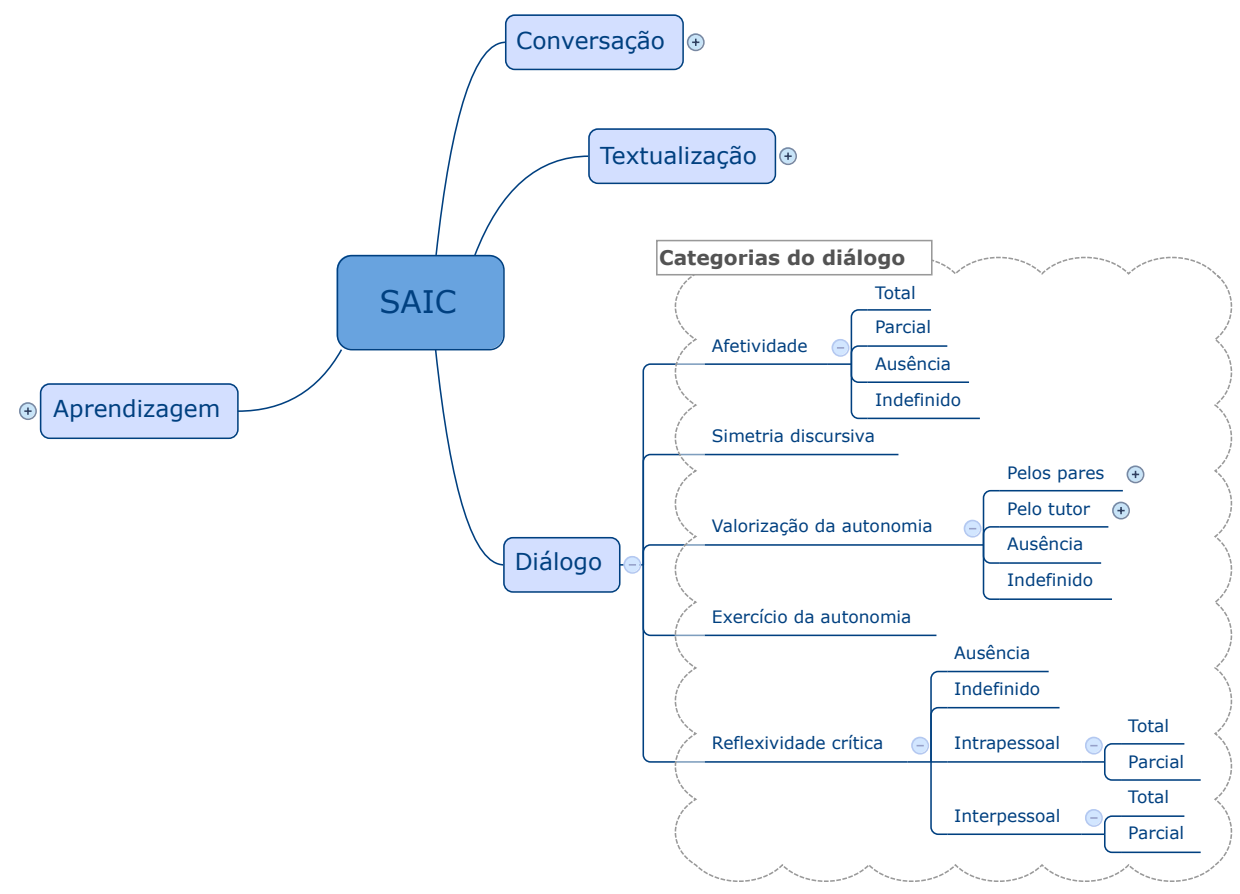

Figura 1. As categorias do SAIC, com especial foco para a categoria Diálogo

A classificação das mensagens nestas categorias é feita a partir da análise de seu conteúdo. Esta análise é feita baseada na identificação de marcadores linguísticos. Por exemplo, se os termos "parabéns" (marca de elogio) e "obrigado" (marca de gratidão) forem encontrados na mensagem, pode-se dizer que existe a presença do parâmetro Afetividade. Tais marcadores foram analisados, identificados, agrupados e associados aos parâmetros do Diálogo (vide Figura 2), a saber (exemplos entre parêntesis):

\footnotetext{
${ }^{2}$ As características particulares dos parâmetros simetria discursiva e exercício da autonomia ainda estão em formulação e, por isso, não foram detalhadas na Figura 1.
} 
- Afetividade: elogio (“parabéns”), saudação inicial (“bom dia!"), destinatário da mensagem (nomes próprios no início da mensagem), despedida ("até mais"),gratidão (“obrigado"), concessão ("perdão") e concordância ("realmente");

- Valorização da autonomia: elogio ("parabéns"), estímulo ("isso mesmo"), proposições/sugestões ("sugiro que"), solicitação de explicação ("por que"), e propostas de questões desafiadoras/problematização ("não achas?”);

- Reflexividade crítica: afirmações (verbo no presente), opinião pessoal ou avaliação ("penso que"), fundamentação ("como diz o autor"), expressão que anuncia lista de tópicos ("como por exemplo:"), referências (citações entre aspas) e presença de conector discursivo de caráter argumentativo ("além disso").

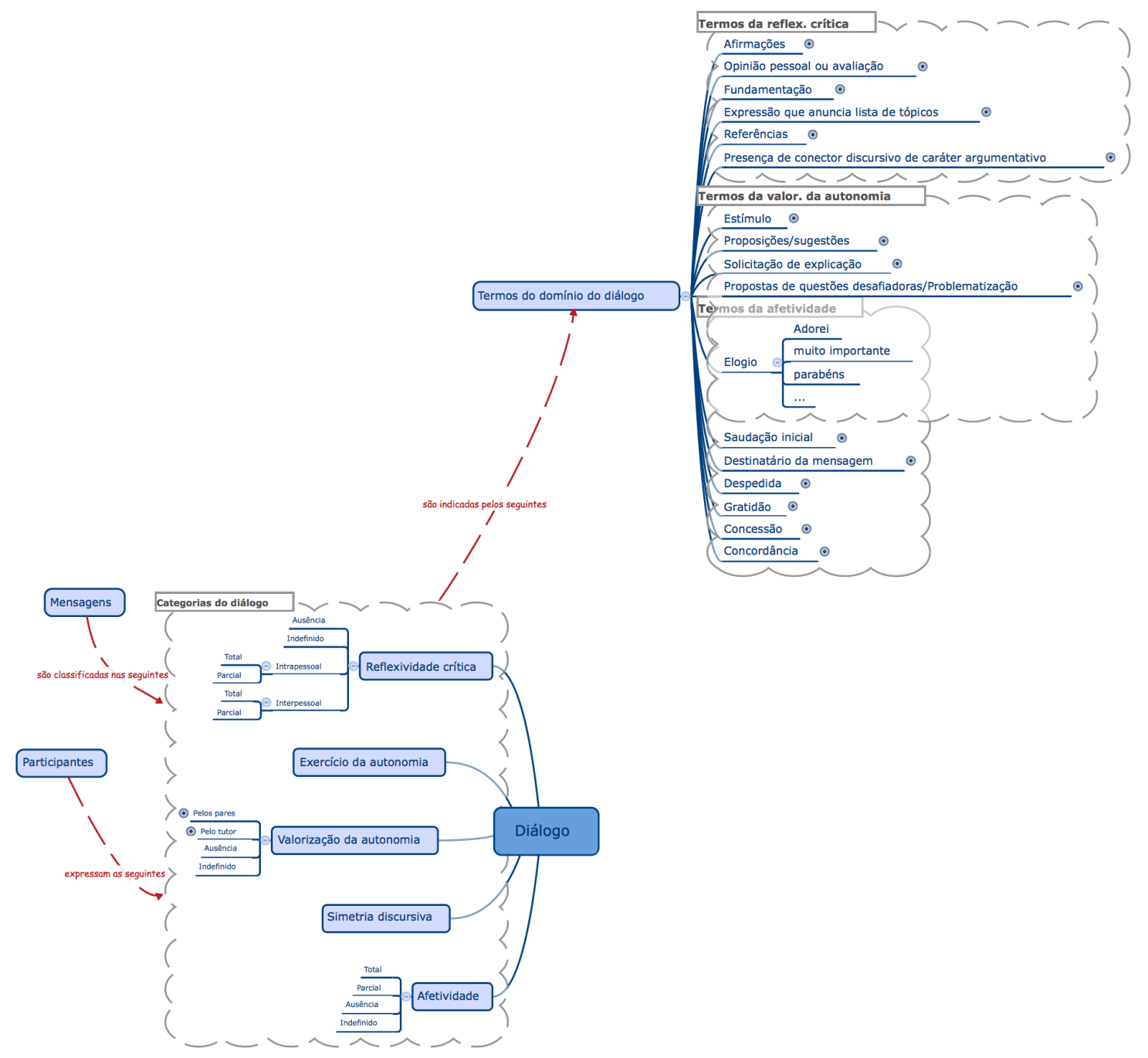

Figura 2. A categoria Diálogo e suas relações com os termos do domínio identificados

A seguir, será apresentada a formalização do domínio Diálogo na ferramenta de modelagem ontológica. 


\subsection{A Representação da Ontologia do Diálogo}

A ontologia do Diálogo foi construída com o auxílio da ferramenta Protégé ${ }^{3}$. Nesta construção, os mapas conceituais serviram de base para o estabelecimento das classes ontológicas e suas relações. Via de regra, cada conceito do mapa foi transformado em uma classe da ontologia. Foram estabelecidas, ainda, relações entre estas classes, como a de subclasse, ou seja, a especialização de um conceito do domínio Diálogo. Por exemplo, os parâmetros do diálogo (Afetividade, Simetria Discursiva, etc.) foram modeladas como subclasses da classe Diálogo. Este mesmo princípio foi utilizado para modelar os marcadores do diálogo. Nesta versão inicial, foram derivadas 54 classes, ilustradas parcialmente na Figura 3.

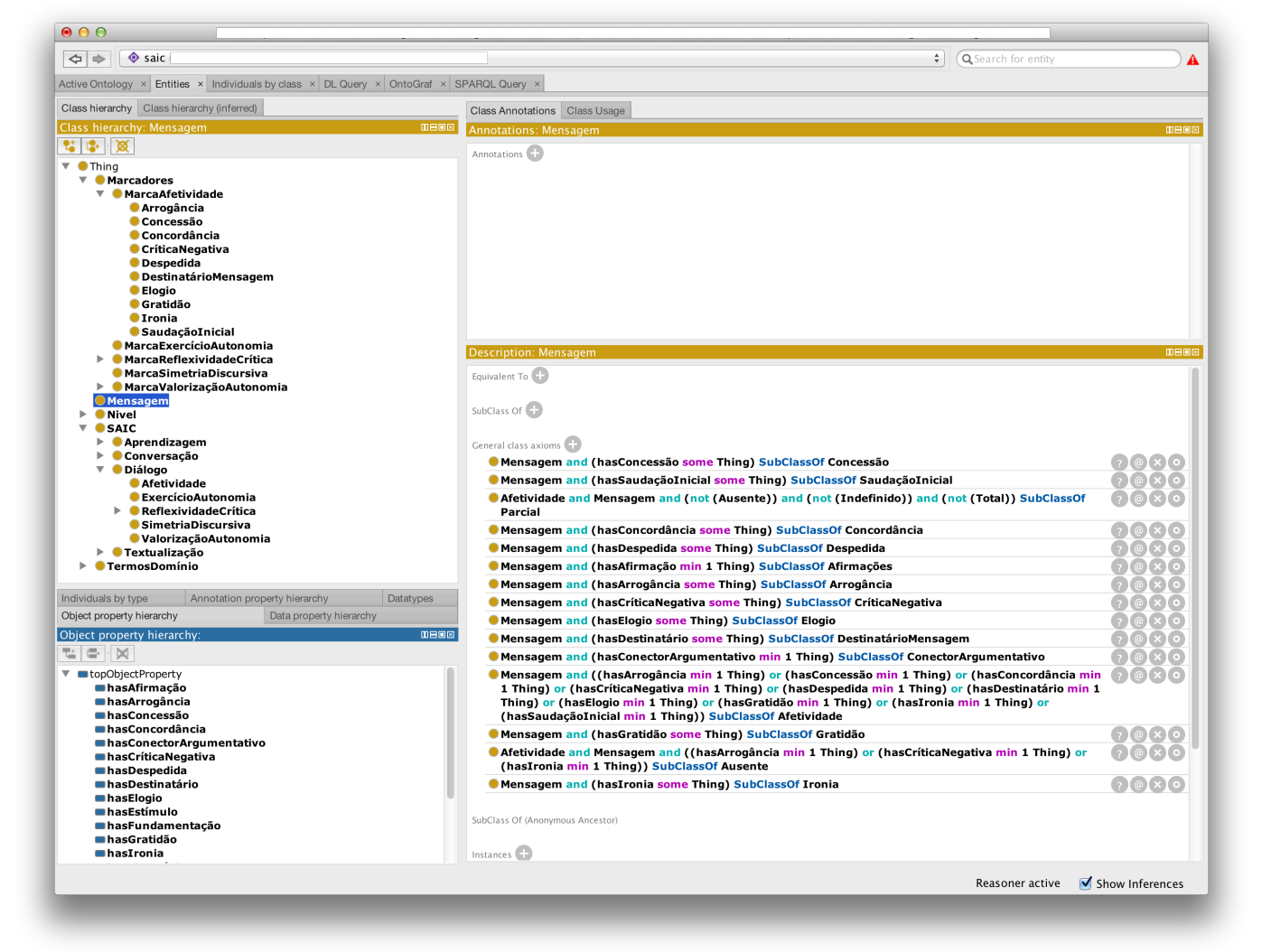

Figura 3. Visão geral das classes da ontologia criada

No SAIC, uma mensagem pode conter diferentes parâmetros simultaneamente (Afetividade e Valorização da Autonomia, por exemplo). Com o intuito de descrever este fato, foram estabelecidas 20 propriedades (object properties) que relacionam uma mensagem com os diferentes marcadores de determinado parâmetro.

Estes parâmetros podem ainda possuir diferentes níveis. Por exemplo, uma mensagem pode possuir Afetividade Total, outra, Afetividade Parcial e uma terceira, Ausência de Afetividade. Tais níveis foram modelados na ontologia por meio de axiomas, como o apresentado na Figura 4. Nele, expressa-se o fato de uma mensagem possuir ausência de afetividade quando apresenta um dos seguintes marcadores: arrogância, crítica negativa ou ironia.

\footnotetext{
${ }^{3}$ http://protege.stanford.edu
} 


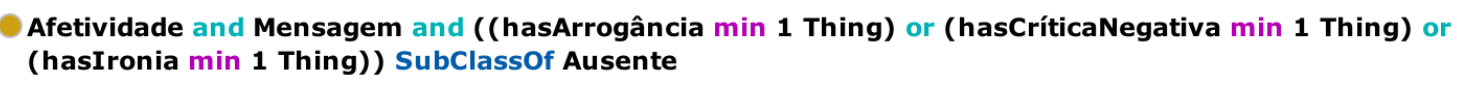

Figura 4. Axioma que representa a classificação de uma mensagem como possuindo ausência de afetividade

Os resultados alcançados com esta formalização representam um avanço frente aos estudos anteriores, descritos na Seção 2 deste artigo, seja por ampliar a compreensão do domínio Diálogo freireano no contexto da Educação a Distância, seja pela viabilização de sua análise automatizada em AVA.

\section{Considerações Finais}

Com o objetivo de prover um maior detalhamento e formalização do domínio Diálogo freireano e tendo em vista implementá-lo em AVA para posterior análise automatizada de mensagens em fóruns de discussão, este artigo apresentou o processo de desenvolvimento da ontologia do Diálogo.

O trabalho teve início com uma ampla revisão da literatura em busca de marcadores linguísticos que identificassem a manifestação dos parâmetros do Diálogo freireano, traduzidos em parâmetros de análise, conforme Omitido para submissão (2009). Os marcadores foram, então, sistematizados em regras capazes de classificar as mensagens de acordo com sua adequação a cada parâmetro. Em seguida, procedeu-se com a formalização do conhecimento, o que gerou o principal resultado do estudo: uma versão inicial da ontologia do diálogo freireano. A estrutura da ontologia foi representada na forma de mapa conceitual e no editor de ontologias Protégé.

Dentre as contribuições do estudo, é possível destacar também uma melhor compreensão sobre a manifestação do diálogo freireano em fóruns de discussão da EaD, a partir do conhecimento de diversos marcadores que ilustram a presença de cada parâmetro do diálogo nas mensagens. Por outro lado, a construção de uma ontologia deste domínio pavimentou o caminho para a formalização das demais categorias do SAIC (Conversação, Textualização e Aprendizagem), o que favorecerá a sua posterior implementação em AVA. Isto permitirá, também, uma ampla validação do SAIC, ampliando significativamente os resultados obtidos com os estudos anteriores, já que estes tiveram um alcance restrito, no que tange o corpus de análise, dada a dificuldade de se analisar manualmente um volume elevado de mensagens.

Como trabalhos futuros, destacam-se: em primeiro lugar, terminar por completo a construção da ontologia do Diálogo (incluindo-se os parâmetros Simetria Discursiva e Exercício da Autonomia) e integrá-la ao AVA Omitido para submissão. A ideia é que o AVA forneça ao professor-tutor um panorama de como as interações entre os alunos estão ocorrendo, ou seja, se as mensagens estão provendo o Diálogo de maneira adequada. Findada esta primeira etapa, pretende-se, ainda, ampliar a ontologia para contemplar todas as outras categorias do SAIC, com semelhante integração ao AVA mencionado.

\section{Agradecimentos}

Os autores agradecem ao CNPq pelo financiamento desta pesquisa. 
CBIE-LACLO 2015

Anais do XXVI Simpósio Brasileiro de Informática na Educação (SBIE 2015)

\section{Referências}

Brasil. (2014). Instituto Nacional de Estudos e Pesquisas Educacionais Anísio Teixeira (INEP). Resumo Técnico: Censo da Educação Superior de 2013. Brasília: Ministério da Educação. Disponível em: http://download.inep.gov.br/educacao_superior/censo_superior/apresentacao/2014/coleti va_censo_superior_2013.pdf.

David, P. B.; Castro-Filho, J. A. (2009). Dialogicidade em práticas interativas da área de exatas. In: Anais do XX Simpósio Brasileiro de Informática na Educação, Florianópolis-SC.

David, P. B. (2010). Interações contingentes em ambientes virtuais de aprendizagem. Tese (Doutorado em Educação) - Universidade Federal do Ceará, Faculdade de Educação, Programa de Pós-Graduação em Educação Brasileira, Fortaleza-CE.

David, P. B.; Castro-Filho, J. A. (2010). Sistema de Análise de Interações Contingentes Aplicado a um Chat Pedagógico. In: Anais do XXI Simpósio Brasileiro de Informática na Educação, João Pessoa-PB.

Freire, R. S.; David, P. B.; Oliveira, F. K. (2011). Dialogicidade na Formação Online de Professores de Matemática. In: Anais do XXII Simpósio Brasileiro de Informática na Educação, Aracaju-SE.

David, P. B.; Castro-Filho, J. A. (2012). Sistema de Análise de Interações Contingentes: Uma Contribuição para Práticas Interativas em Cursos a Distância. Revista eCurriculum (PUCSP), v. 8, p. 1-28.

David, P. B.; Freire, R. S. ; LIMA, J. G. M. (2013a). Dialogicidade na formação online de professores de português. In: Anais do X Congresso Brasileiro de Ensino Superior a Distância. Porto Alegre: Associação Universidade em Rede-UniRede. v. 1.p. 1-15.

David, P. B.; Freire, R. S. (2013b). A Abordagem Freireana na Formação Online de Professores-Tutores. In: XVIII Conferência Internacional sobre Informática na Educação, Porto Alegre-RS.

Freire, P. (2006). Pedagogia do Oprimido. Rio de Janeiro: Paz e Terra. $1^{\text {a }}$ Edição: 1970.

Fontes, L. M. O.; Mendes-Neto, F. M.; Pontes, A. A. A. (2011). OntoPBL: Uma Ontologia de Domínio sobre Aprendizagem Baseada em Problema. In: XXII Simpósio Brasileiro de Informática na Educação - SBIE, Aracaju.

ICDE. (2013). International Council for Open and Distance Education Strategic Plan 2013-2016. Disponível http://www.icde.org/filestore/About/ICDEStrategicPlan2013-16website.pdf

Isotani, S.; Mizoguchi, R. (2010). Planejamento e Análise de Sessões Collaborativas Utilizando Teorias de Aprendizagem e Ontologias. Revista Brasileira de Informática na Educação, v. 15, n.2, p. 45-56.

Noy, N. F.; Mcguinness, D. L. (2001). Ontology Development 101: A Guide to Creating Your First Ontology. Stanford Knowledge Systems Laboratory Technical Report KSL-01-05 e Stanford Medical Informatics Technical Report SMI-20010880 . 